\title{
Medición del ángulo vestíbulo-lingual en conductos mesiales de primeros molares inferiores utilizando tomografía de haz cónico.
}

\author{
Measuring the vestibular-lingual angle in lower molar- \\ first mesial ducts using cone beam tomogiraphy.
}

\author{
Alfonso Espinosa Torres,* Kaori E Galaso Trujillo,* Alejandra Barrón Aguilar,* Humberto Alejandro Monreal Romero ${ }^{\ddagger}$
}

\section{RESUMEN}

El objetivo del presente estudio fue medir la curvatura vestíbulo-lingual de los conductos de las raíces mesiales de primeros molares inferiores en personas adultas del estado de Chihuahua, por medio de la técnica de tomografía computarizada de haz cónico (CBCT, por sus siglas en inglés) y usando el software de análisis 3D Endo ${ }^{\mathrm{TM}}$ (Dentsply/Sirona USA). Se llevó a cabo la medición por dos observadoras, empleando dos métodos diferentes, Schneider y 3D Endo ${ }^{\mathrm{TM}}$ (Dentsply/Sirona USA). Los resultados obtenidos tuvieron valores mayores a los 100 grados con la técnica 3D Endo ${ }^{\mathrm{TM}}$ y a los 20 grados con la técnica de Schneider. No se detectó una diferencia estadística significativa al comparar los diferentes conductos entre sí.

Palabras clave: Ángulo vestíbulo-lingual, molar inferior, CBCT, 3D Endo $^{\mathrm{TM}}$.

\section{ABSTRACT}

The objective of the present study was to measure the vestibule-lingual curvature of the ducts of the mesial roots of lower first molars, of adults from the state of Chihuahua. Using the cone beam computed tomography (CBCT) technique and using the $3 D$ analysis software Endo $^{T M}$ (Dentsply/Sirona USA). The measurement was carried out by two observers using two different methods, Schneider and $3 D$ Endo ${ }^{T M}$ (Dentsply/Sirona USA). The results obtained had values greater than 100 degrees with the $3 D$ Endo ${ }^{T M}$ technique and at 20 degrees with the Schneider technique. No statistically significant difference was detected when comparing the different ducts with each other.

Keywords: Vestibulo-lingual angle, lower molar, CBCT, $3 D$ Endo ${ }^{T M}$.

\section{INTRODUCCIÓN}

$\mathrm{D}^{\mathrm{e}}$ ebido a la complejidad anatómica radicular, es muy importante conocer las diferentes variantes anatómicas de los canales radiculares para un adecuado diagnóstico y tratamiento endodóntico. La morfología de los canales radiculares puede variar entre poblaciones étnicamente diferentes. ${ }^{1}$

Las variables anatómicas de los dientes multirradiculares en ocasiones dan como resultado el fracaso del tratamiento de conductos. En la actualidad, con la ayuda de microscopio endodóntico, la tomografía de haz cónico (CBCT, por sus siglas en inglés) y diversos softwares como el 3D Endo ${ }^{\text {TM }}$ (Dentsply/Sirona USA) podemos identificar y visualizar la trayectoria de los conductos en estos dientes, así como su localización respecto a diversas estructuras anatómicas antes y durante el abordaje endodóntico. ${ }^{2}$

Schneider fue el primero en establecer un método seguro y de confianza para poder determinar ese grado de curvatura a partir de vistas radiográficas clínicas, pero no investigó desde la vista proximal. ${ }^{3}$

\footnotetext{
* Profesor.

¥ Profesor Investigador.

Facultad de Odontología de la Universidad Autónoma de Chihuahua. México.

Recibido: 07 de julio de 2021. Aceptado: 11 de enero de 2022.
}

Citar como: Espinosa TA, Galaso TKE, Barrón AA, Monreal RHA. Medición del ángulo vestíbulo-lingual en conductos mesiales de primeros molares inferiores utilizando tomografía de haz cónico. Rev ADM. 2022; 79 (1): 12-19. https://dx.doi.org/10.35366/103813

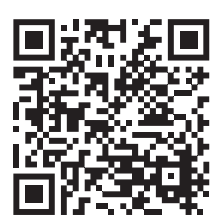


Conocer el grado de curvatura del canal radicular es esencial para el momento de seleccionar el tipo de instrumento para trabajar, así como la técnica de instrumentación. Minimizando el impacto y dificultades anatómicas, así como sus limitaciones de instrumentos de endodoncia, previniendo consecuencias desastrosas durante la preparación del conducto, tales como la pérdida de la longitud de trabajo, transportación apical, creación de escalones y perforaciones, o incluso la fractura de los instrumentos. . $^{4-7}$

La CBCT es una herramienta auxiliar en el diagnóstico que nos permite observar una pieza dental por medio de una reconstrucción tridimensional elaborada por computadora, además permite observar diferentes planos axial, sagital y coronal. Con esta herramienta podemos evaluar las diferentes y complejas anatomías a las que nos enfrentamos, tales como número y forma de los canales radiculares y raíces, longitud de éstas, así como las diferentes curvaturas que puedan presentar en diferentes estudios, se han aprovechado estas características para analizar la anatomía de los órganos dentarios..$^{8-13}$

Existen diversos equipos de software, como el 3D Endo $^{\mathrm{TM}}$ (Dentsply/Sirona USA) que nos simplifican la interpretación de estas CBCT y nos ayudan a realizar un plan de tratamiento más eficaz, con este software en particular podemos hacer un trazo de la dirección de las curvaturas (vestibular o palatino) de los canales radiculares y así poder observar con más claridad su trayecto y curvaturas. ${ }^{14}$

El presente estudio se realizó con la finalidad de conocer el grado de curvatura radicular en sentido vestíbulolingual que se presenta en las raíces mesiales de primeros molares inferiores en un grupo poblacional de México.

\section{MATERIAL Y MÉTODOS}

Para realizar este estudio, se recabó información de diversos centros de radiodiagnóstico en la provincia de
Chihuahua, México de CBCT de primeros molares inferiores de pacientes a los que se les habían realizado estos estudios por motivos ajenos al presente trabajo.

La población total de primeros molares inferiores que se recolectó de los centros de radiodiagnóstico fue de 50 molares.

Para establecer el tamaño de muestra, se consideró un intervalo de confianza de 95\% y un error estándar de $5 \%$. Para calcular el tamaño de la muestra se utilizó el software es.surveymonkey.com, el cual arrojó un resultado de 45 molares, siendo este número el que se utilizó como tamaño de la muestra.

Se utilizaron estudios de tomografía de haz cónico tomados a pacientes que contaban por lo menos con un primer molar inferior y que cumplían con los siguientes criterios de inclusión: dientes permanentes, sin tratamiento endodóntico previo, con ápices completamente formados, con dos conductos en su raíz mesial, que no presenten fisuras o fracturas radiculares, que no presenten indicios de resorción interna o externa, espesor de corte de la CBCT menor a $0.300 \mathrm{~mm}$, resolución de la CBCT inferior a $0.300 \mathrm{~mm}$, FOV de $5 \times 5$.

Se llevó a cabo la medición de la curvatura de los conductos mesiales de cada muestra utilizando dos métodos, el primero fue la técnica de Schneider y para el segundo se utilizó el programa 3D Endo ${ }^{\text {TM }}$ (Dentsply/ Sirona USA).

Para poder observar cada muestra, se requirió separar digitalmente las imágenes tomográficas de cada una de ellas del resto de los componentes anatómicos de cada paciente visibles en la tomografía, para ello, por medio del programa 3D Endo ${ }^{\mathrm{TM}}$ (Dentsply/Sirona USA), se recortó la imagen de cada molar en los planos axial, coronal y sagital (Figura 1).

Una vez individualizado cada molar, se procedió a trazar la trayectoria corono-apical, tanto del conducto mesio-bucal (MB), como del mesio-lingual (ML), para ello se empleó la herramienta de selección de conductos del

Figura 1: Molar inferior previo

al corte con 3D EndoTM. A)

Vista coronal. B) Vista sagital.
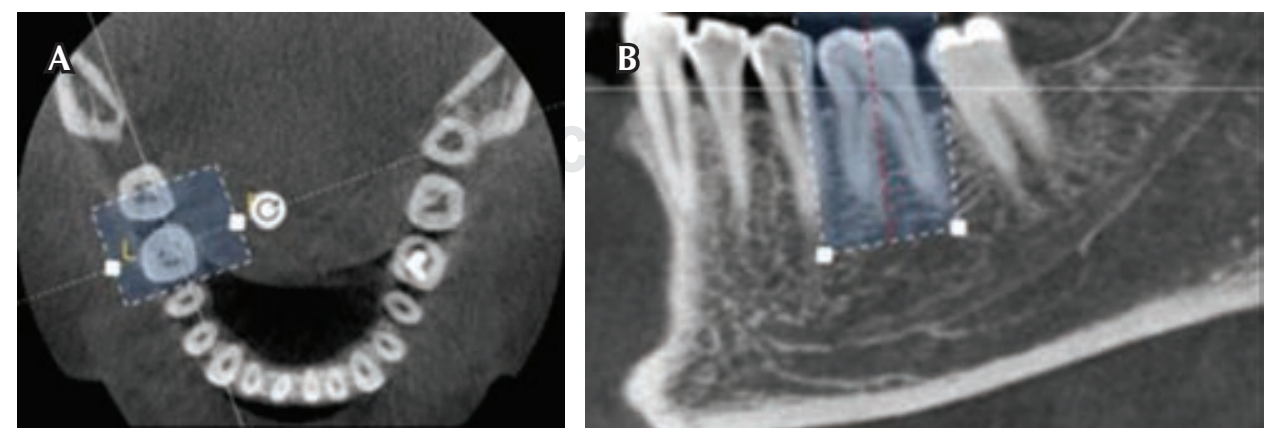

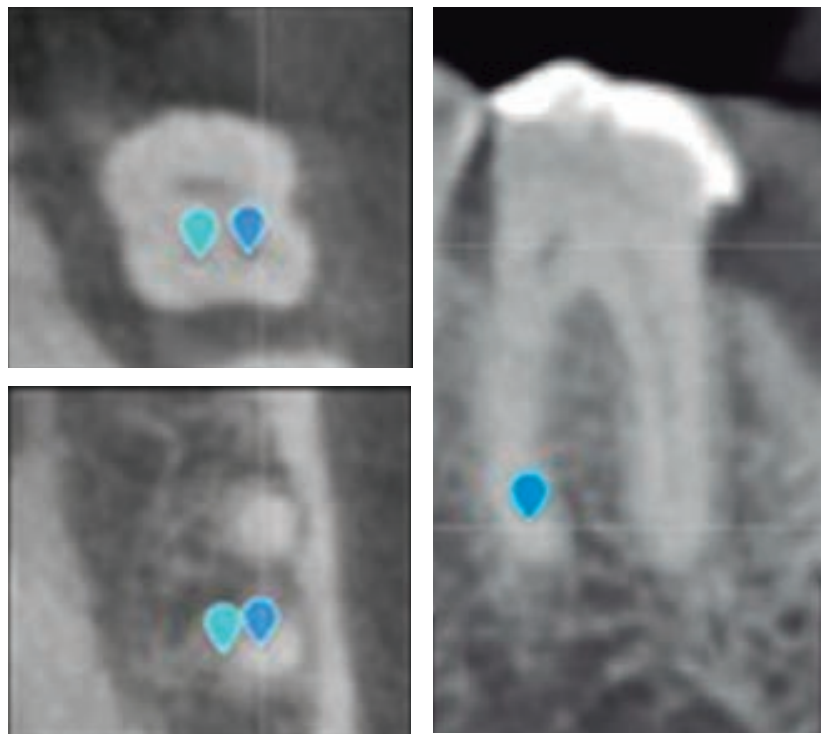

Figura 2: Marcación de los conductos mesiales en la porción coronal y apical.
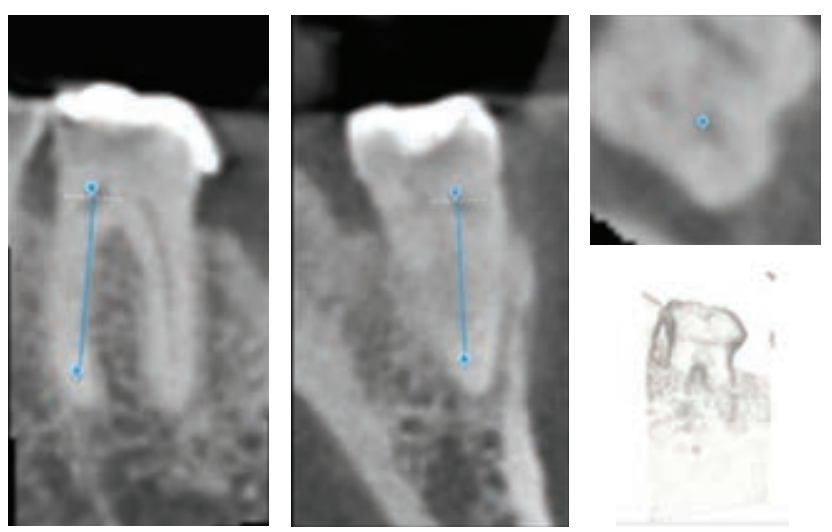

Figura 3: Trazo inicial de unión de los extremos coronal y apical.

software, por medio de esta herramienta se ubican marcas con forma de gota invertida, tanto en la zona coronal como apical de ambos conductos, el programa ofrece colores diferentes de estas figuras para identificarlas, la posición de estas marcas se controla visualmente en forma simultánea, en los planos coronal, sagital y axial, la colocación de cada marca puede ajustarse de forma manual (Figura 2).

Una vez hecho lo anterior, el software identifica la trayectoria de cada conducto y el operador puede elegir cuál de los dos conductos desea observar, al seleccionar un conducto, el programa presenta una pantalla con imá- genes de éste en sentido axial, sagital y coronal, además de una reconstrucción de todo el molar en 3D (Figura 3).

Estas imágenes pueden ajustarse a voluntad del operador para corregir variaciones en la trayectoria en sentidos
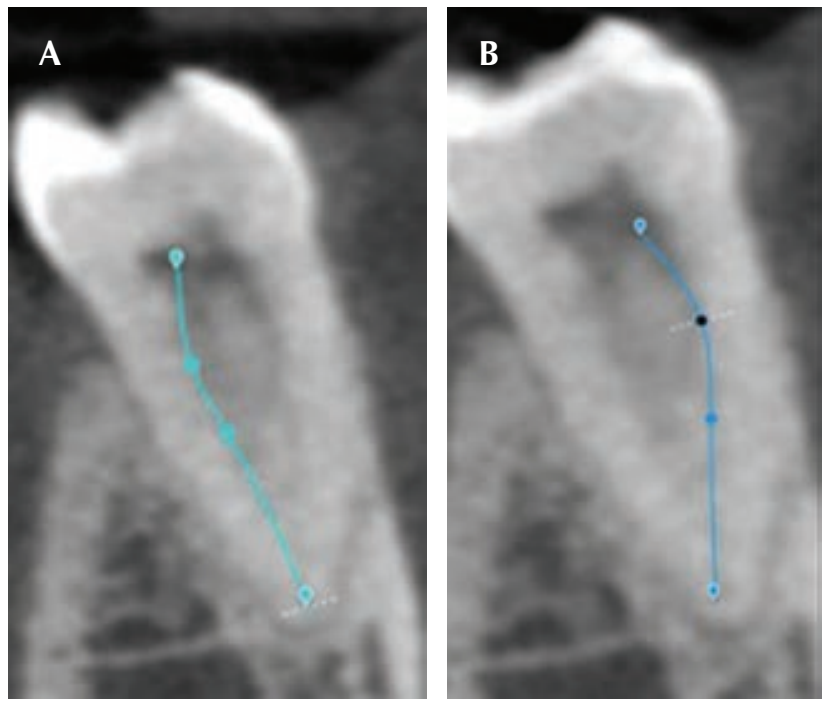

Figura 4: Ajuste de la trayectoria: A) conducto mesio-bucal y B) conducto mesio-lingual.
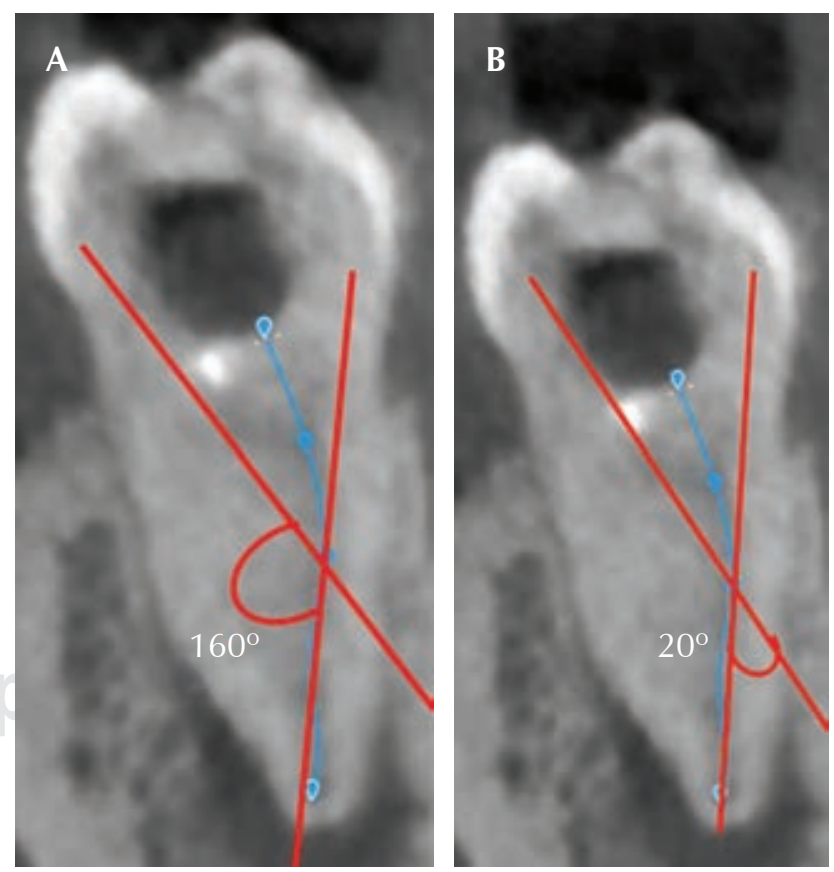

Figura 5: Trazado del ángulo de la curvatura. A) Técnica 3D Endo ${ }^{\mathrm{TM}}$. B) Técnica Schneider. 

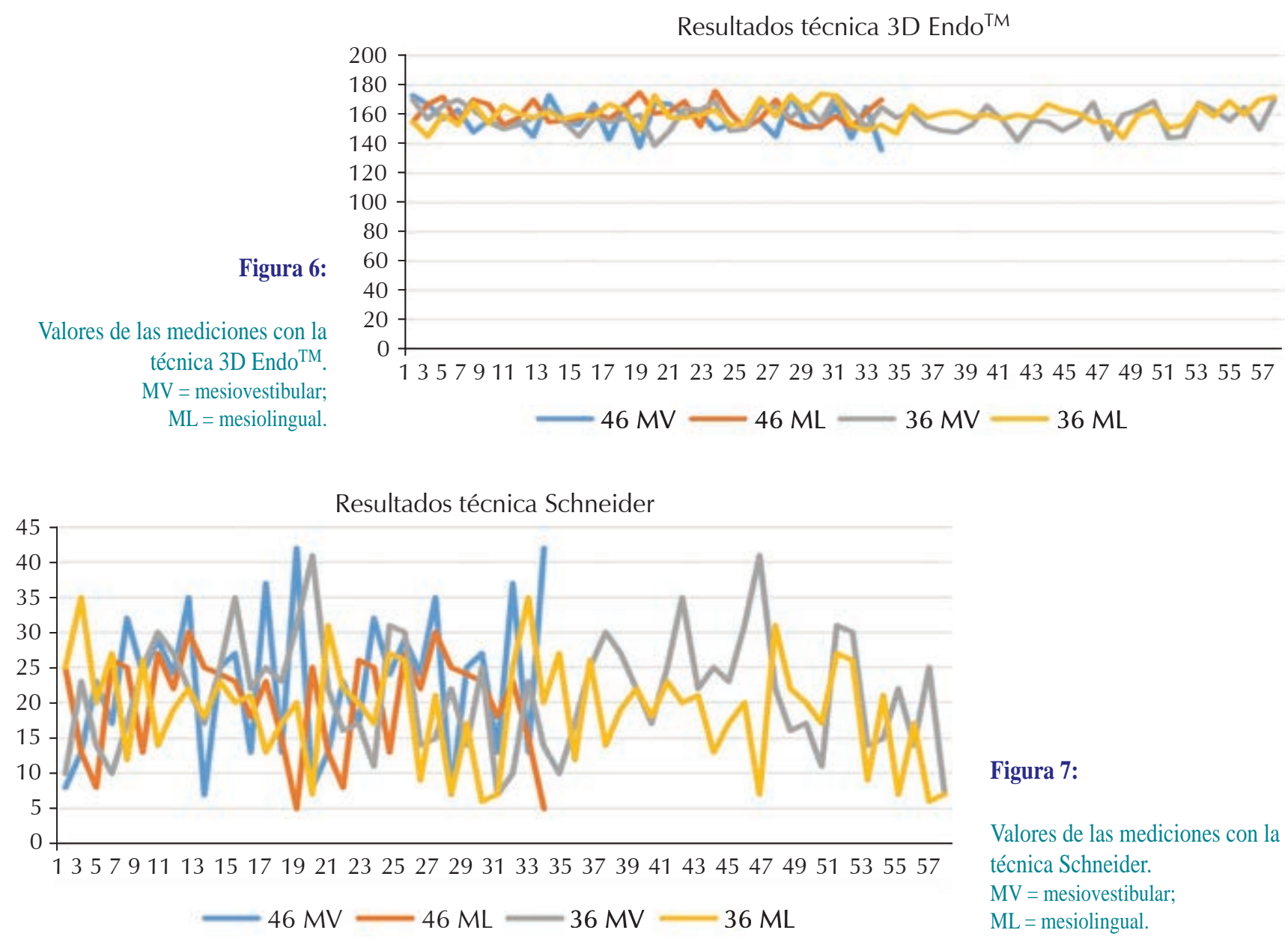

axial y sagital (Figura 4), en el sentido coronal se puede seguir la trayectoria del trazo desplazando la imagen para verificar que se encuentre centrada con relación al canal correspondiente.

Una vez que se estableció la trayectoria final de cada conducto, la imagen correspondiente se almacenó en una carpeta con los datos de número de diente y conducto (bucal o lingual) según correspondía.

Para realizar la medición de la curvatura, cada imagen se imprimió en papel bond y se presentó a dos de los investigadores, quienes hicieron la medición del ángulo de cada conducto utilizando un transportador simple (Figura 5).

Cada observador realizó una doble medición de cada conducto, una por medio de la técnica de Schneider y otra con la técnica de 3D Endo ${ }^{\mathrm{TM}}$ que consiste en seguir el trazo del canal en sentido corono-apical hasta la primera curvatura, de ahí desplazarse siguiendo el trayecto trazado del canal hasta el ápice.

\section{RESULTADOS}

Las mediciones obtenidas por ambas observadoras se recopilaron en una hoja de Excel, para su análisis se elaboraron gráficas con estos datos, las gráficas muestran una mayor uniformidad de los resultados obtenidos con la técnica 3D Endo ${ }^{\mathrm{TM}}$ (Figura 6), así como una mayor variación en los resultados obtenidos con la técnica de Schneider (Figura 7) en ambas observadoras.

Para conocer si existe diferencia entre los valores angulares de los diferentes conductos y técnicas se utilizó la prueba t Student en la hoja de cálculo Excel, el resultado mostró que no se presentó diferencia estadísticamente significativa al comparar los conductos mesiales en cada diente, ni al compararlos individualmente con sus contralaterales tanto con la técnica $3 \mathrm{D} \mathrm{Endo}^{\mathrm{TM}}$ como con la técnica Schneider.

De manera complementaria a las mediciones de las curvaturas de los conductos mesiovestibular (MV) y 
mesiolingual $(\mathrm{ML})$, en las imágenes de $\mathrm{CBCT}$ se pudo detectar que existen muestras que presentan una doble curvatura generalmente en el conducto $M L$, estas curvaturas se encontraron mayormente en la unión de los tercios medio-coronal y medio-apical respectivamente (Figura 8), en estos casos la mayoría de los conductos MV presentaban una sola curvatura.

Además de lo anterior, también se pudo constatar que un alto porcentaje de las muestras presentaba una unión de los conductos MV y ML en el tercio apical, lográndose identificar en algunas de estas muestras una zona radiolúcida de forma triangular en el mencionado tercio apical (Figura 9), la observación de esta zona triangular se facilita utilizando el programa 3D Endo $^{\mathrm{TM}}$,

Figura 8:

Trazo del conducto mesiolingual de diente 36 con doble curvatura.

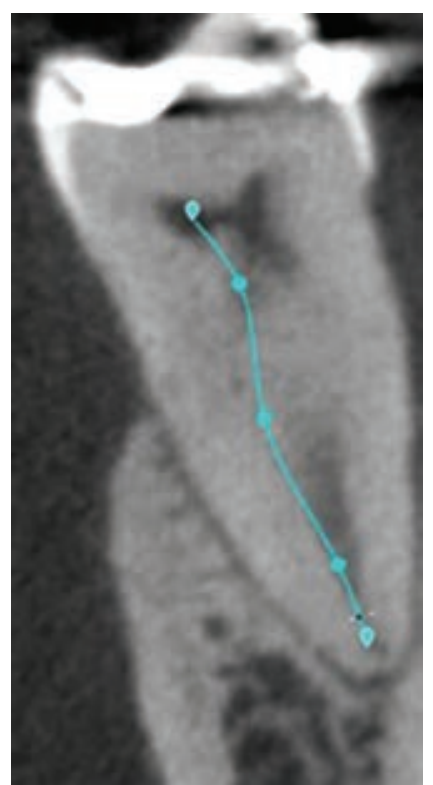

gracias a que permite orientar en forma lateral el ángulo de visión de las imágenes de la raíz, lo que permite una observación simultánea de los conductos MV y ML en la proyección axial.

Por otra parte, en las muestras donde no se observaba unión de los conductos MV y ML en el tercio apical, las curvaturas de éstos tendían a ser más regulares y abiertas (Figura 10). En este caso, la proyección coronal de la CBCT muestra ambos conductos separados hasta la zona apical sin presencia de istmo.

Un ejemplo de lo anterior se puede observar por medio de la técnica de pulpectomía lateral ${ }^{15}$ que muestra la posición de limas dentro de conductos $\mathrm{MV}$ y $\mathrm{ML}$ con salidas independientes en apical (Figura 11).

Otra forma de observar la posición de las limas en el interior de conductos MV y ML es con la reconstrucción $3 \mathrm{D}$ que realiza en forma automática del programa 3D Endo $^{\text {TM }}$ (Figura 12).

\section{DISCUSIÓN}

La anatomía de los primeros molares inferiores, específicamente de los conductos mesiales en sentido vestíbulolingual, presenta morfologías radiculares con alto grado de complejidad, en las cuales se destacan curvaturas abruptas, lo que puede condicionar a accidentes en el tratamiento de conductos, tales como: fractura de instrumentos, pérdida de la longitud de trabajo, transportación apical, creación de escalones y perforaciones; lo anterior se ha reportado en diferentes estudios, nuestra investigación concuerda con lo mencionado anteriormente, ya que las muestras estudiadas presentaban curvaturas vestíbulo-linguales de diversa complejidad. ${ }^{16-19}$

Diversos estudios señalan que la morfología radicular está ligada al tipo de población y/o grupo étnico, ${ }^{20-25}$
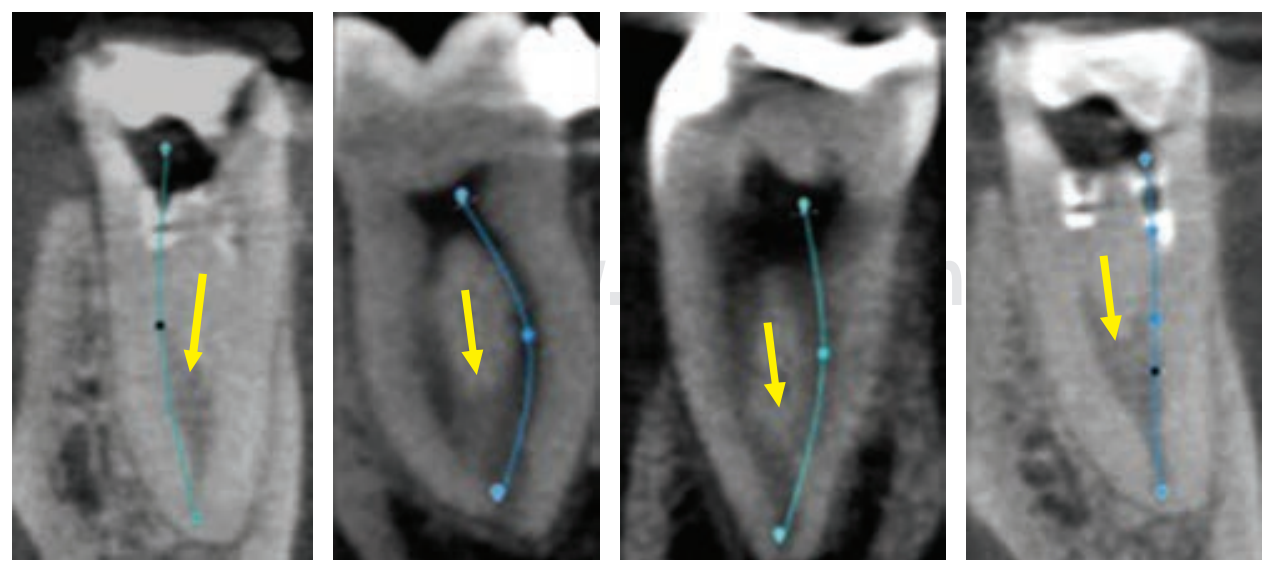

Figura 9: Zonas radiolúcidas de forma triangular en tercio apical presentes en las muestras donde se unían los conductos mesiovestibular y mesiolingual. 

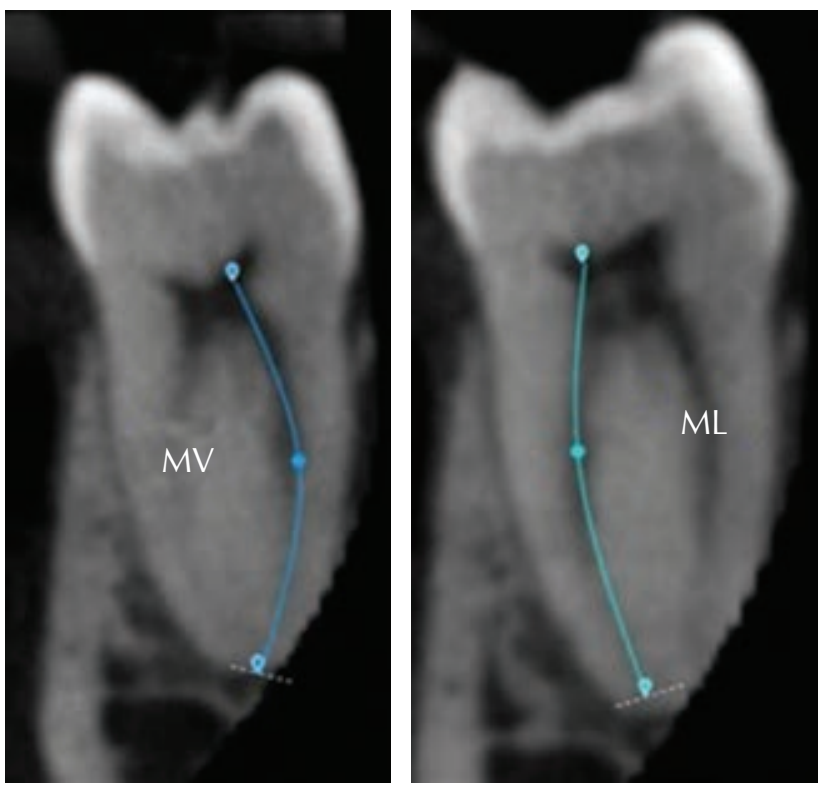

Figura 10: Conductos mesiovestibular (MV) y mesiolingual (ML) en un diente 36 que no se unen en el foramen apical, sus curvaturas son más uniformes y abiertas.

por lo que surge la inquietud de realizar este trabajo de investigación, enfocado en la población del estado de Chihuahua. Si bien no tenemos aún parámetros en la población mexicana para comparar con nuestro estudio, al tener nuestro país diferentes grupos étnicos podemos deducir que existen también diferentes morfologías en los conductos radiculares.

En la presente investigación la disposición de los conductos presentó 22 de las muestras $(48.8 \%)$ con clase tipo II de Vertucci, 14 muestras con el tipo III (31.11\%) y nueve muestras con el tipo IV (20\%), a diferencia de estudios previos realizados, en los cuales el mayor porcentaje de las clases de Vertucci se encuentra de la siguiente manera: primero clase IV (52.0\%), seguida de la clase II (28.7\%). ${ }^{26}$

Para la medición de los ángulos, se estableció que fueran dos investigadores del estudio quienes se encargaran de realizar las mediciones de todas las muestras de manera individual para disminuir el riesgo de sesgo y/o error. Respecto a la diferencia de las angulaciones entre los conductos mesiovestibulares y mesiolinguales, los resultados de esta investigación demuestran que cuando se realizó la medición con la técnica de Schneider por ambas observadoras, coincide con otros estudios donde se muestra que el mayor grado de curvatura es predominante en el conducto mesiovestibular y con mayor frecuencia en el tercio apical. ${ }^{17}$
Asimismo, nuestra investigación reporta que la curvatura vestíbulo-lingual puede no ser tan abrupta, pero si sumamos a ésta la curvatura mesiodistal en la unión del tercio apical de los conductos mesiovestibular y mesiolingual, encontramos diversos factores como se menciona en otras investigaciones que pueden llevar a que la instrumentación de los conductos mesiales de los molares inferiores sea complicada, generando torsiones y estrés de los instrumentos endodónticos, aumentando el riesgo de accidentes transoperatorios. ${ }^{17}$

De igual forma, coincidimos con estudios anteriores que mencionan que la radiografía convencional o digital es únicamente un auxiliar en el diagnóstico, siendo insuficiente para la identificación completa de las estructuras anatómicas y patológicas. ${ }^{4}$

En la actualidad se puede utilizar la ayuda de sistemas computarizados enfocados en el área de endodoncia para el análisis de tomografías, con los cuales se puede obtener la mayor exactitud en el procedimiento clínico al conocer la morfología radicular, en este trabajo se determinó usar el software para el análisis del trayecto radicular 3D Endo ${ }^{\mathrm{TM}}$ (Dentsply Sirona), cuyos resultados concuerdan con resultados de otros investigadores que también lo emplearon para visualizar de manera más precisa la morfología radicular, lo que ha permitido obtener imágenes completas y detalladas tanto del conducto mesiovestibular como del mesiolingual, y así poder emplear dos técnicas de medición angular de las curvaturas vestíbulo-linguales en estos conductos; de igual manera,

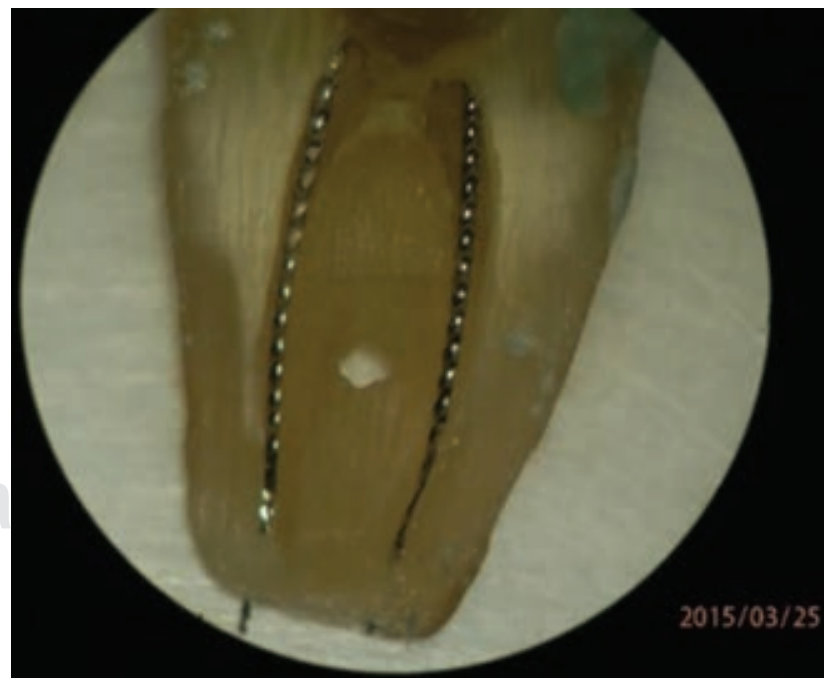

Figura 11: Micrografía 16× de raíz mesial del primer molar inferior con conductos separados. 
Figura 12:

Vista sagital de la ubicación de limas en conductos mesiovestibular y mesiolingual unidos en apical reconstrucción en 3D. dental convencional, esto pretende ayudar a disminuir los accidentes durante esta fase del tratamiento, seleccionando de una manera más adecuada la técnica de instrumentación en estos procedimientos clínicos.

Dado que no se obtuvo diferencia estadística al comparar los resultados de los valores angulares y las técnicas de medición utilizadas, podemos establecer que existe cierta uniformidad anatómica en los conductos mesiovestibulares de primeros molares inferiores. La curvatura vestíbulo-lingual, al tener una configuración relativamente uniforme, puede influir o no en la dificultad de los tratamientos de endodoncia.

\section{AGRADECIMIENTOS}

Los autores agradecen al Centro de Radiodiagnóstico FACE IMAGEN de la ciudad de Chihuahua las facilidades para la recolección de la muestra del presente estudio.

\section{REFERENCIAS}

tivo en la entodoncia moderna, minimizando errores en la práctica clínica en cuyos casos la anatomía radicular presenta cierta complejidad. 6,15,20-25,27-36

El no encontrar diferencia significativa en los resultados puede ser una muestra de cierto grado de simetría anatómica entre los conductos mesiovestibular y mesiolingual, aunque eso no significa que existan casos donde las variantes anatómicas entre ellos sean mayores; esto puede tener correlación con estudios de caracterización morfológica donde se ha observado simetría bilateral en la morfología oclusal de molares inferiores. ${ }^{37}$

\section{CONCLUSIONES}

La tecnología CBCT y 3D Endo ${ }^{\mathrm{TM}}$ (Dentsply Sirona) en forma combinada demostraron ser de gran utilidad para identificar y medir el grado de las curvaturas vestíbulolinguales en raíces mesiales de molares inferiores, permitiendo una observación directa de los conductos radiculares en forma simultánea y completa. Los ángulos de las curvaturas superaron lo considerado en la hipótesis al promediar más de 100 grados medidos con la técnica 3D Endo ${ }^{\mathrm{TM}}$ (Dentsply Sirona) y más de 20 grados con la técnica Schneider, salvo el promedio de los conductos mesiolinguales del diente 36 que fue de 18 grados.

Se demostró que, aunado a los factores ya existentes en relación con las dificultades para instrumentar los conductos mesiales de los primeros molares inferiores, se debe agregar la presencia de la curvatura vestíbulolingual, la cual no se puede observar en una radiografía
1. Ravishankar Verma G, Bhadage C, Bhoosreddy AR, Vedpathak PR, Mehrotra GP, Nerkar AC et al. Cone beam computed tomography study of root canal morphology of permanent mandibular incisors in indian subpopulation. Pol J Radiol. 2017; 7 (82): 371-375.

2. Di Nardo D, Gambarini G, Costantini R, Testarelli L, Piasecki L, Al-Sudani D et al. 3D clinical evaluation of unusual anatomy of a maxillary second molar: a case report. Srp Arh Celok Lek. 2018; 147 (7-8): 475-478.

3. Cunningham CJ. A three-dimensional study of canal curvatures in the mesial roots of mandibular molars. J Endod. 1992; 18 (6): 294-300.

4. Hartmann RC, Fensterseifer M, Peters OA, de Figueiredo JAP, Gomes MS, Rossi-Fedele G. Methods for measurement of root canal curvature: a systematic and critical review. Int Endod J. 2019; 52 (2): 169-180.

5. Estrela C, Bueno MR, Sousa-Neto MD, Pécora JD. Method for determination of root curvature radius using cone-beam computed tomography images. Braz Dent J. 2008; 19 (2): 114-118.

6. Van der Vyver PJ, Paleker F, Vorster M. 3D software, glide path management and gold-wire instruments: setting the stage for treating complex root canal anatomy. Int Dent South Africa. 2018; 8 (4): 16-30.

7. Schneider SW. A comparison of canal preparations in straight and curved root canals. Oral Surg Oral Med Oral Pathol. 1971; 32 (2): 271-275.

8. Kim I, Paik KS, Lee SP. Quantitative evaluation of the accuracy of micro-computed tomography in tooth measurement. Clin Anat. 2007; 20 (1): 27-34.

9. Scarfe WC, Farman AG, Sukovic P. Clinical applications of conebeam computed tomography in dental practice. J Can Dent Assoc. 2006; 72 (1): 75-80.

10. Álvarez CE, Nazar PM, Caro AM. Anatomía de molares. Trabajo de investigación seminario I. Valparaíso: Universidad de Valparaíso; 2013.

11. Kim SY, Kim BS, Woo J, Kim Y. Morphology of mandibular first molars analyzed by cone-beam computed tomography in a Korean 
population: Variations in the number of roots and canals. J Endod. 2013; 39 (12): 1516-1521.

12. Espinosa Torres A, Colín HI, Espinosa CV. Estudio del ángulo de curvatura mesio-distal en conductos vestibulares de primeros molares superiores empleando CBCT y $3 \mathrm{~d}$-endo en un grupo de población mexicana. Endodoncia Militar. 2019; 3 (3): 354-361.

13. Vertucci FJ. Root canal morphology and its relationship to endodontic procedures. Endod Top. 2005; 10 (1): 3-29.

14. Gambarini G, Ropini P, Piasecki L, Costantini R, Carneiro E, Testarelli $L$ et al. A preliminary assessment of a new dedicated endodontic software for use with CBCT images to evaluate the canal complexity of mandibular molars. Int Endod J. 2018; 51 (3): 259-268.

15. Espinosa-Torres A. Pulpectomía lateral. Una técnica de microodontología para desarrollar habilidad en el manejo de microscopios dentales. Rev ADM. 2014; 71 (2): 95-99.

16. Plotino G, Grande NM, Melo MC, Bahia MG, Testarelli L, Gambarini G. Cyclic fatigue of NiTi rotary instruments in a simulated apical abrupt curvature. Int Endod J. 2010; 43 (3): 226-230.

17. Lee JK, Yoo YJ, Perinpanayagam H, Ha BH, Lim SM, Oh SR et al. Three-dimensional modelling and concurrent measurements of root anatomy in mandibular first molar mesial roots using microcomputed tomography. Int Endod J. 2015; 48 (4): 380-389.

18. Aminsobhani $M$, Sadegh $M$, Meraji N, Razmi $H$, Kharazifard MJ. Evaluation of the root and canal morphology of mandibular permanent anterior teeth in an Iranian population by cone-beam computed tomography. J Dent (Tehran). 2013; 10 (4): 358-366.

19. Altunsoy M, Ok E, Nur BG, Aglarci OS, Gungor E, Colak M. A conebeam computed tomography study of the root canal morphology of anterior teeth in a Turkish population. Eur J Dent. 2014; 8 (3): 302-306.

20. Soleymani A, Namaryan N, Moudi E, Gholinia A. Root canal morphology of mandibular canine in an iranian population: a CBCT assessment. Iran Endod J. 2017; 12 (1): 78-82.

21. Fu Y, Deng Q, Xie Z, Sun J, Song D, Gao Y et al. Coronal root canal morphology of permanent two-rooted mandibular first molars with novel 3D measurements. Int Endod J. 2020; 53 (2): 167-175.

22. Ordinola-Zapata R, Bramante CM, Versiani MA, Moldauer BI, Topham G, Gutmann JL et al. Comparative accuracy of the Clearing Technique, CBCT and Micro-CT methods in studying the mesial root canal configuration of mandibular first molars. Int Endod J. 2017; 50 (1): 90-96.

23. Schryvers A, Govaerts D, Politis C, Lambrechts P. Endodontic management of a maxillary first molar with two palatal roots: $\mathrm{A}$ case report. Aust Endod J. 2019; 45 (3): 420-425.

24. Shah PB, Shah N, Kariya P. Software guided predictable endodontic management of three-rooted lower right second premolar. Int J Prev Clin Dent Res. 2018; 5: 81-83. Available in: http://www.ijpcdr.org

25. Kim K, Choi HS, Pang NS. Clinical application of 3D technology for tooth autotransplantation: a case report. Aust Endod J. 2019; 45 (1): 122-128.

26. Tomaszewska IM, Skinningsrud B, Jarzebska A, Pekala JR, Tarasiuk J, Iwanaga J. Internal and external morphology of mandibular molars: An original micro-CT study and meta-analysis with review of implications for endodontic therapy. Clin Anat. 2018; 31 (6): 797-811.

27. Tchorz JP, Wrbas KT, Von See C, Vach K, Patzelt SBM. Accuracy of software-based three-dimensional root canal length measurements using cone-beam computed tomography. Eur Endod J. 2019; 4 (1): 28-32.

28. Tchorz JP. 3D Endo: three-dimensional endodontic treatment planning. Int J Comput Dent. 2017; 20 (1): 87-92.

29. Gaudin A, Pérez F, Galicia J. Digital technology in endodontics. In: Digital restorative dentistry. Springer International Publishing; 2019. pp. 229-247.

30. Lang T, Gaengler P. Guided endodontic treatment using a new software approach-case report [Internet]. [Cited 2019 Nov 18]. Available in: www.siriusendo.de

31. He S, Kau CH, Liao L, Kinderknecht K, Ow A, Saleh TA. The use of a dynamic real-time jaw tracking device and cone beam computed tomography simulation. Ann Maxillofac Surg. 2016; 6 (1): 113-119.

32. Tchorz JP, Wrbas KT, Hellwig E. Guided endodontic access of a calcified mandibular central incisor using a software-based threedimensional treatment plan. Int J Comput Dent. 2019; 22 (3): 273281. Available in: http://www.ncbi.nlm.nih.gov/pubmed/31463491

33. Shi $X$, Zhao $S$, Wang $W$, Jiang $Q$, Yang X. Novel navigation technique for the endodontic treatment of a molar with pulp canal calcification and apical pathology. Aust Endod J. 2018; 44 (1): 66-70.

34. Mordanov O, Khabadze Z, Daurova F, Bagdasarova I, Zoryan A, Kulikova A et al. Second mesiobuccal canal evaluation features with cone-beam computed tomography. Int J Dent. 2019; 2019: 5856405. doi: 10.1155/2019/5856405.

35. Maggiore C, Gallottini L, Resi JP. Mandibular first and second molar. The variability of roots and root canal system. Minerva Stomatol. 1998; 47 (9): 409-416.

36. Plotino G, Tocci L, Grande NM, Testarelli L, Messineo D, Ciotti M, et al. Symmetry of root and root canal morphology of maxillary and mandibular molars in a white population: a cone-beam computed tomography study in vivo. J Endod. 2013; 39 (12): 1545-1548.

37. García A, Gustín P, Quiñonez C, Sacanamboy L, Torres MH, Triana $L$ et al. Morphological characterization of incisors and molars of an African descent group of Cali, Valle del Cauca (Colombia). Rev Estomat Salud. 2015; 23 (2): 17-29.

Conflicto de intereses: Los autores declaran no tener conflicto de intereses.

Aspectos éticos: No aplica.

Financiamiento: El financiamiento corrió por cuenta de los autores.

Correspondencia:

Alfonso Espinosa Torres

E-mail: alfonso.endo@hotmail.com 